Tyrer P, Tyrer H (2017b) Health Anxiety: Part 2 - Cognitive Behaviour Therapy (CPD Online Module). Royal College of Psychiatrists.

Tyrer P, Salkovskis P, Tyrer H, et al (2017c) Cognitive-behaviour therapy for health anxiety in medical patients (CHAMP): randomised controlled trial with outcomes to 5 years. Health Technology Assessment, 21(50): 1-58.
Van den Heuvel OA, Veale D, Stein DJ (2014) Hypochondriaisis: considerations for ICD-11. Revista Brasiliera de Psiquiatria, 36: S21-7.

Walker J, Vincent N, Furer P, et al (1998) Treatment preference in hypochondriasis. Journal of Behavioural and Therapeutics in Experimental Psychiatry, 30: 251-8.

\section{MCOs}

Select the single best option for each question stem

1 You suspect that a patient in your clinic has excessive health anxiety. What is the next question you put to them?

a Have you any mental health problems?

b How many times have you consulted over this trouble?

c Do you think the investigations you have had are really necessary?

d Do you worry a lot about your health?

e Have people ever suggested you are a hypochondriac?

2 A patient comes to you with a complaint of pain in the chest, but investigation finds no organic cause. When is health anxiety the most likely explanation for the presentation?

a When the patient admits they are anxious about the pain

b When the patient has had a recent heart attack c When the patient checks their heart rate repeatedly

d When the pain is continuous

e When the pain is not helped by analgesics.

3 Antidepressant drug treatment is of limited benefit in health anxiety because:

a most patients are not depressed

b it takes too long before positive changes are noticed

c the dose has to be raised to high levels to be effective

d to drug effects tolerance develops very quickly

e health-anxious patients are very nervous about the possible adverse effects of drugs.

4 The prevalence of health anxiety is highest in:

a primary care

b psychiatric out-patient clinics

c out-patient clinics in medical secondary care, d accident and emergency departments

e in-patients.

5 Patients with health anxiety respond well to cognitive-behavioural therapy when they realise that:

a they have excessive worry and fear of disease

b they are under the age of 40

c they no longer need reassurance

d they trust what the doctor tells them

e they have to die of something.

\title{
coRseEsoum Working beyond your job plan
}

\section{Alex Till \& Jan Wise}

doi: 10.1192/apt.bp.117.017293, Published by Royal College of Psychiatrists, November 2017.

In the article by Till (2017), an error occurred in the author information for Jan Wise. The author details are reproduced below.

Jan Wise is a consultant psychiatrist with Central and North West London NHS Foundation Trust. He chairs and sits on several BMA committees involved in trade union duties, caring for doctors so that they can care for patients.

\section{Reference}

Till A, Wise J (2017) Working beyond your job plan. BJPsych Advances Nov 2017, 23 (6) 395-396; D0I: 10.1192/apt.bp.117.017293 